\title{
Characterization of molecular pathways for targeting therapy in glioblastoma
}

\author{
Naveed Wagle, Minhdan Nguyen, Jose Carrillo, Judy Truong, Lucia Dobrawa, Santosh Kesari \\ John Wayne Cancer Institute, Santa Monica, CA, USA \\ Contributions: (I) Conception and design: N Wagle; (II) Administrative support: None; (III) Provision of study materials or patients: None; (IV) \\ Collection and assembly of data: N Wagle; (V) Data analysis and interpretation: N Wagle; (VI) Manuscript writing: All authors; (VII) Final approval \\ of manuscript: All authors. \\ Correspondence to: Naveed Wagle, MD. John Wayne Cancer Institute, 2200 Santa Monica, CA 90404, USA. Email: WagleN@jwci.org.
}

\begin{abstract}
Glioblastoma remains the most common malignant brain neoplasm in adults. The available therapies for treatment have only modestly extended survival. Traditional chemotherapy agents have shown only slight effectiveness in controlling this disease. The use of molecular profiling has allowed personalized medicine options to be explored for the care of these individuals. Targeted therapies have shown significant benefit in numerous other cancer types with survival being extended significantly. In glioblastoma, several promising markers have been identified including vascular endothelial growth factor (VEGF), epidermal growth factor receptor (EGFR), and programmed cell death protein 1 (PD-1)/programmed death-ligand 1 (PD-L1). These targets have been shown to play a critical role in glioblastoma formation and proliferation. The pathways of these receptors have been elucidated in detail. This level of understanding has led to the a more robust understanding of possible mechanism of pathway modification. The targeting of these specific markers has led to the development of several selective therapies with additional therapies being evaluated. The clinical trials validating these markers have been promising but have yet to show a clear benefit in brain tumors. This identification of alternative methods to address these markers or identify additional targets may be the key to the fight against this disease. The molecular targeting of glioblastoma pathways may have significant impact on disease control and patient survival.
\end{abstract}

Keywords: Glioblastoma; targeted; glioma; epidermal growth factor receptor (EGFR); vascular endothelial growth factor (VEGF); programmed cell death protein 1 (PD-1)

Submitted Mar 20, 2020. Accepted for publication Dec 16, 2020.

doi: $10.21037 / \mathrm{cco}-20-124$

View this article at: http://dx.doi.org/10.21037/cco-20-124

\section{Introduction}

The most common malignant primary brain neoplasm is adults is glioblastoma. The World Health Organization (WHO) classifies this as a grade four neoplasm. Glioblastoma has a median life expectancy of approximately 12 months despite current therapies. Radiation and concurrent temozolomide chemotherapy remains the standard of care initial therapy after surgery (1). This treatment regimen has had a significant impact on progression-free survival (PFS) and overall survival. Novo-TTF, a non-pharmaceutical intervention, has also shown improved outcomes in glioblastoma treatment (2).
Bevacizumab, although received accelerated approval for the treatment of recurrent glioblastoma, efficacy continues to remain unclear. Novel and more precise advances have provided new optimism.

Chemotherapy has traditionally focused non-specific mechanisms to prevent cell growth, this focused heavily on the inhibition of DNA replication. The mechanisms tumors can replicate and survive has been robustly explored. The understanding of molecular basis for cancer proliferation has changed our treatment strategy to specific molecularly targeted therapies. Potentially, tumors with aberrant signaling in one pathway are more likely to respond to 
agents targeting that pathway. Agents that target a different pathway would like less effect in treating this tumor. This is described personalized medicine in that treatment is targeted to molecular profiles unique to the cancer. Intense research into clinical trials that are no aimed at determining these molecularly targeted agents which may benefit patients with glioblastoma (3).

\section{Standard therapy for glioblastoma}

Surgical resection is the first step in the diagnosis and treatment of glioblastoma. Subsequently, standard protocol includes radiation therapy with concurrent temozolomide. Patients are subsequently treated with adjuvant temozolomide therapy for a minimum of 6 cycles. This standard therapy has shown an overall survival of 14.6 months with a median PFS of 6.9 months and a $25.6 \% 2$-year survival rate (1). These lackluster findings demonstrate that glioblastoma continues to have a grim prognosis. The treatment for recurrent glioblastoma has not standard of care option as no clear efficacious therapy has emerged. Traditional chemotherapies have long been evaluated without a significant impact on survival. The most frequently used therapies include, carmustine, carboplatin, irinotecan, BCNU wafers, or repeat surgical intervention. These recurrent treatment options have minimal survival impact. However, without any clear alternatives they remain the most widely used options to date.

\section{Genetic variations in glioblastoma}

Many mutations have been shown to have a role in the development of glioblastoma. The Tumor Cancer Genome Atlas (TCGA) has shown three main pathways activated in most glioblastoma tumors. These are the epidermal growth factor receptor (EGFR), retinoblastoma (RB), and p53 pathways (4). Numerous clinical trials have attempted to address the components of these pathways but none have shown any clinical efficacy (3). The development of an effective targeted therapy has been challenging given the heterogeneity in glioblastoma. Bevacizumab remains the only targetable therapy which has shown significant response and possible clinical efficacy.

EGFR is an upstream receptor that is activated by the binding of epidermal growth factor. In glioblastoma, there is often a ligand-independent mutation of the receptor, called EGFRvIII, which is constitutively active (5-14). The activation of this receptor results in recruitment of phosphoinositide 3-kinase (PI3K) to the cell membrane. Phosphatidylinositol (PI)-4,5-bisphosphate is phosphorylated to PI-3-phosphate (PIP3) by PI3K. PIP3 subsequently activates downstream molecules such as protein kinase B (AKT) and mTOR (15). Cell proliferation and inhibition of apoptosis is the result of this signaling cascade. Phosphate and tensin homology (PTEN) serves as a checkpoint in this system by inhibiting PIP3 signaling. It also has homology to the catalytic region of protein tyrosine phosphates which are important to the function of PIP3. The gene for PTEN is located at $10 \mathrm{q} 23.3$. In $15-40 \%$ of glioblastomas there is mutation of this gene which supports the assumption that deregulation of this pathway is common is glioblastoma $(12,16,17)$. EGFR signal transduction also stimulates Ras proteins. Ras proteins are membrane associated GTPases which require post-translational addition of a farnesyl group to the C-terminus. This is accomplished by farnesyl transferase (18). Ras proteins, once activated, stimulate cellular proliferation, survival and angiogenesis (19). The Ras protein and farnesyl transferase are potential targets for inhibiting the EGFR pathway.

The $\mathrm{p} 53$ pathway is mutated in $87 \%$ of glioblastomas (4). The transformation of lower grade gliomas to glioblastoma is proposed to involve mutation in the $\mathrm{p} 52$ pathway. TP53 stimulates apoptosis or senescence as a response to DNA damage. The TP53 gene is located at chromosome 17 p13.1 and causes loss of regulatory control if mutation or homozygous deletion occurs (20). Murine double minute 2 (MDM2), which inhibits cells from entering apoptosis, binds TP53 blockings its ability to activate transcription of promoter sequences (21). This in turn essentially may cause glioblastoma cells to gain immortality by amplification of MDM2. The AFR gene product lies upstream of MDM2 which can bind to it. ARF binding of MDM2 inhibits the function of p53 (22-24). Homozygous deletion or promoter methylation of this gene product has been found frequently in glioblastoma leading to loss of expression. Promotor methylation is seen both primary glioblastoma and glioblastoma which as transformed form a lower grade glioma (secondary glioblastoma) (25). Disruption of any of these MDM2, TP53, or ARF products can cause cells to lose normal function because there is a strong autoregulatory feedback between these genes.

The $\mathrm{RB}$ protein is required for progression through the cell cycle. Mutations in the RB pathway have been found in $78 \%$ of glioblastomas (4). The RB protein is required for the cell to progress form G1 phase to $S$ phase. RB inhibits $\mathrm{E} 2 \mathrm{~F}$ transcription factor which activates genes 
involved with transition between phases (26). The RB protein is phosphorylated by the CDK4/CCND2/CDK6 complexes which inhibits it activity. CDK4/CCND2/ CDK6 amplification is seen in $1-18 \%$ of glioblastomas. Cell replication increase as a result of this amplification. P16 and CDKN2B act to inhibit these complexes and lie upstream of these complexes. Alteration in the expression of any of these genes, RB, or CDK4 complexes can lead to uncontrolled cell division.

Glioblastoma therapeutics are now focused on the development of targets for many of these genes, proteins, pathways, and complexes. It is known that certain subgroups of glioblastoma patients respond divergently to targeted treatment options. Glioblastoma cells have variable molecular genetic patterns that confer aberrant abilities to replicate cells. While multiple pathways may be present in a glioblastoma often times there appears to be dominant process. If a targeted agent to affect this dominant pathway it would have a significant impact of tumor growth and proliferation. This effect would be limited to the subgroup that showed this pathway and not effective in subgroup that was driven by an alternative dominant pathway. Thus, the need for personalized medicine and the use of molecularly targeted interventions.

\section{Vascular endothelial growth factor (VEGF) molecular targeted therapy}

Glioblastoma is known to shown increased VEGF production. VEGF stimulates vascular proliferation in the area surrounding glioblastoma cells. The result is increased nutrient supply, oxygen delivery, and blood flow. Glioblastoma growth and proliferation is enhanced by these factors. It has been shown that high levels of VEGF in gliomas are associated with a worse prognosis (27).

Bevacizumab was the first targeted therapy approved for the treatment of glioblastoma. Bevacizumab is a humanized monoclonal antibody against VEGF. Bevacizumab inhibits the VEGF signaling pathway preventing increased vascular supply to glioblastoma cells. The Avaglio study was a phase 3 randomized controlled study investigating the use of this agent in newly diagnosed glioblastoma patients. In this study 458 patients received bevacizumab while 463 patients received placebo. The study revealed a median PFS of 10.6 months in the bevacizumab group versus 6.2 months in the placebo group [stratified hazard ratio for progression or death, $0.64 ; 95 \%$ confidence interval (CI), 0.55 to $0.74 ; \mathrm{P}<0.001]$ (28). However, overall survival did not differ significantly between the two groups (stratified hazard ratio for death, $0.88 ; 95 \% \mathrm{CI}, 0.76$ to $1.02 ; \mathrm{P}=0.10$ ) (28). Overall survival at one year with bevacizumab was $72.4 \%$ and placebo was $66.3 \%(\mathrm{P}=0.049)$ Overall survival was respectively $33.9 \%$ and $30.1 \%$ at 2 years $(\mathrm{P}=0.24)(28)$. The bevacizumab group maintained health-related quality of life and performance status longer. They also had a lower glucocorticoid requirement. There was in increased incidence of grade 3 or higher adverse events in the bevacizumab group versus the placebo group $(66.8 \%$ versus $51.3 \%$ ) (28). This large study shows the addition of bevacizumab to standard of care with radiotherapy and temozolomide did not improve survival in patients with glioblastoma. The patients had a significantly longer PFS and maintain a higher performance status and quality of life in the bevacizumab arm. They also had a higher incidence of adverse events.

Despite these results the targeting of VEGF continues to be a pathway of intense optimism. The PFS benefits seen in the phase 3 trial and reports in numerous other publications keep bevacizumab a frequently used option in recurrent glioblastoma. The use of combination therapies with bevacizumab and traditional chemotherapy options is also being explored. New VEGF targeting agents have also started to enter clinical trials.

\section{Programmed cell death protein 1 (PD-1)/ programmed death-ligand 1 (PD-L1) checkpoint blockage}

The PD-1/PD-L1 pathway suppresses the function of T cells in removing tumor cells (29-31). Several types of solid tumors have shown upregulation of PD-L1. Tumors that shows a high expression levels of PD-L1 often have better outcomes with PD-1/PD-L1 checkpoint blockades (32-35). These findings lead to the evaluation of PD-L1 expression and the clinical efficacy of checkpoint blockades in glioblastoma patients.

Newly diagnosed glioblastoma has increased PD-L1 expression in $88 \%$ of specimens. Recurrent glioblastoma specimens $72.2 \%$ of probability of PD-L1 overexpression (36). One study showed that $61 \%$ glioblastoma patients had PD-L1 overexpression (37). The principal was tested in the Checkmate 143 clinical trial. The trial investigated the use of nivolumab, PD-L1 blocking agent with or without ipilimumab, a cytotoxic T-lymphocyte-associated antigen 4 (CTLA-4) blocking agent. The phase 1 cohort of the study evaluated the clinical effects and tolerability in addition 
to the safety of nivolumab with or without ipilimumab in patients with recurrent glioblastoma. Forty patients in total where evaluated. Ten patients were randomized to receive nivolumab as a single agent, ten patients received low-dose nivolumab and low-dose ipilimumab, and the other twenty patients received high-dose nivolumab and high-dose ipilimumab (38). The subgroup receiving nivolumab alone tolerated the treatment better than other two subgroups (38). Fatigue and diarrhea were the most common treatment-related adverse events. The study was also found that the dose of the higher dose ipilimumab was correlated with poorer patient tolerance (38). The poor tolerance may be explained because ipilimumab has an earlier impact on $\mathrm{T}$ cell activation. This activation results in an extensive impact in the immune network (38).

Checkmate 143 proceed onto a phase three clinical trial. The study compared nivolumab with bevacizumab therapy in recurrent glioblastoma patients who had failed standard of care upfront therapy with radiation and temozolomide (39). This study revealed nivolumab alone did not have an impact overall survival. The median PFS was 1.5 months for nivolumab versus 3.5 months for bevacizumab (39). The median overall survival was 9.8 months for nivolumab versus 10.0 months for bevacizumab (39). The objective response rate (ORR) has assessed by magnetic resonance imaging was 8 versus 23 months in the nivolumab and bevacizumab arms respectively (39). Lymphopenia caused by radiotherapy was hypothesized as a plausible etiology of the nivolumab monotherapy ineffectiveness. Radiotherapy has been shown to have an effect on circulating lymphocytes decreasing they function (40). Nivolumab monotherapy works by competitively binding $\mathrm{PD}-1$ receptors so a decrease functional lymphocyte count and reduce potential targets. An addition hypothesis for the subdued response of nivolumab is that effector $\mathrm{T}$ cells are anergic to specific antigens. The lymphocytes in gliomas specimens typically possess CD95, PD-1, PD-L1, CTLA-4, lymphocyteactivation gene 3 (LAG3), and T-cell immunoglobulin and mucin domain-containing protein 3 (TIM-3) antigens (41). Tumor infiltrating lymphocytes (TILs) express immunoinhibitory molecules, including CTLA-4 and PD1 , or co-express PD-1 and TIM-3. These targets may be exhausted on $\mathrm{T}$ cell population in tumors $(42,43)$. The $\mathrm{T}$ cell anergy in glioblastoma by we too great for PD-1 checkpoint blockage to overcome on its own. Further studies with combination therapy remain ongoing.

PD-1 and PD-L1 are one of the first targeted therapies to utilize the immune system in the battle against cancer.
This approach has not only advanced targeted options despite the negative results in glioblastoma it has also open the door to other possible immunotherapies. The immune pathway offers a multitude of potential mechanism of action that remain to be explored.

\section{EGFR}

Genetic alterations in receptor tyrosine kinase (RTK) signaling pathways are present in the majority of glioblastomas (44). Among the most relevant pathways is EGFR activation (45). EGFR amplification, mutation, rearrangement, altered splicing or genetic alteration is seen in $57 \%$ of glioblastoma (44-48). The majority of these cases had regional DNA amplification. This led to a wide range of mutation allelic frequencies. Comparing the allelic frequencies of point mutations in DNA- and RNA-seq data revealed a high degree of concordance between the type and prevalence of mutations at the DNA level and the composition of expressed mRNA transcripts. A complete picture of aberrant exon junctions and a semi-quantitative assessment of their expression levels was also provided by RNA-seq (44).

Transcript allelic fraction (TAF) was calculated as the ratio of each aberrant exon junction to the sum of aberrant and wild-type junctions at the 3 ' junction end. In $11 \%$ of tumors, the aberrant exon 1-8 junction characteristic of EGFRvIII was highly expressed. At least $19 \%$ of tumors showed low level expression ( $\geq 1 \%$ ). Digital mRNA assay was able to come to the same conclusion. EGFRvIII expression in glioblastoma is associated with an aggressive tumor phenotype through paracrine mechanism (49).

A variety of other recurrent noncanonical EGFR transcript forms were detected in the RNA-seq data. Three different C-terminal rearrangements targeting the cytoplasmic domain of the EGFR were detected at $\geq 10 \% \mathrm{TAF}$ in $3.7 \%$ of cases and at $\geq 1 \%$ TAF in another $9 \%$. Whole genome sequencing (WGS) data confirmed the presence of $\mathrm{C}$-terminal deletions. These variants have previously been associated with glioma formation in experimental rodent systems in vivo (50). The complete loss of the $\mathrm{C}$ terminus may yield aberrant terminal junctions not able to be mapped by transcriptome sequencing. Under-expression of C terminus exons 27-29 (<3 SD) were apparent in another $7.3 \%$ of cases without detectable aberrant junctions.

Two relatively uncharacterized recurrent EGFR variants, namely deletions of exons $12-13(\Delta 12-13)$ in $28.7 \%$ and 
exons $14-15(\Delta 14-15)$ in $3 \%$ have also been identified. EGFR $\Delta 12-13$ has been previously identified by RT-PCR analysis of glioma (51). Both $\Delta 12-13$ and $\Delta 14-15$ appear to be expressed in minor allelic fractions $(<10 \%)$. This finding raises the question of whether they result from genomic deletion or splicing aberration. WGS analysis of aberrant junctions did not shows a concordant DNA deletion in tumors expressing $\Delta 12-13 \mathrm{mRNA}$. This data suggests concordant breakpoint as a minor component of a highly rearranged locus. By comparison, EGFRvIII-expressing tumors had concordant deletion spanning exons $2-7$ in WGS data (51).

EGFR amplification is frequently associated with a deletion mutation affecting exons $2-7$, referred to as EGFRvIII or delta-EGFR. Twenty to thirty percent of all glioblastomas show EGFRvIII expression $(44,52,53)$. The potential immunogenicity of the EGFRvIII mutation leads to the production of peptide vaccine, rindopepimut, containing the specific novel amino acid sequence created by the EGFRvIII deletion mutation conjugated to keyhole limpet haemocyanin. Rindopepimut was evaluated in two phase 2 trials, ACTIVATE (54) and ACT II (55), as well as a larger phase 2 trial ACT III (56). Collectively, about 100 patients newly diagnosed EGFRvIII expressing glioblastoma treated with a gross total resection and standard of care chemo-radiation were given single agent rindopepimut (ACTIVATE) or rindopepimut plus adjuvant temozolomide (ACT II and ACT III). These trials showed a 15-month PFS and a 24-month overall survival from time of diagnosis $(52,57)$. These results compared favorably with contemporary patient cohorts who received standard treatment. The selection of patients with minimal residual disease in all these trials after completion of chemoradiation assumed that it would minimize the tumor-associated immunosuppression typical of glioblastoma.

The ACT IV study investigated newly diagnosed glioblastoma patient treated with rindopepimut versus a placebo control. The study failed to show an overall survival benefit for either the minimal residual disease or intent to treat populations. Median overall survival in the rindopepimut group was 20.1 versus 20.0 months in the control group (57). In the minimal residual disease population and 17.4 versus 17.4 months, respectively (57). In an exploratory analysis of the stable residual disease population median overall survival was similar between the rindopepimut treatment groups 14.8 versus 14.1 months in the control group (57). These studies investigating the use of an EGFRvIII antagonist did not show a benefit for glioblastoma patients. Direct EGFRvIII antagonism not mediated by an antibody and EGFR antagonist remain to be explored.

\section{Conclusions}

The identification of molecular pathways to cancer formation and treatment has changed the landscape of oncology. The molecular targets identified in glioblastoma have expanded our understanding of the disease process. The VEGF receptor, EGFR, PD-1/PD-L1 pathway have been established is driving mechanisms of glioblastoma formation and growth. Each of these pathways has led to the discovery of multiple potential targets. The targeted therapies for these pathways are aggressively being developed and continue to push into clinical trials. First generation drugs have proceeded to large scale clinical trials. Unfortunately, a clear treatment efficacy remains elusive at this time. The trials each show significant promise but failed to meet set efficacy endpoints. However, the elucidation of these targets has opened the path to the development of new options. There are ongoing trials investigating neurotrophin signaling, BRAF, NTRK, and MET targets currently. These may be used in combination to block more than one pathway in a single tumor. The use of targeted therapies has already changed many oncology treatment paradigms. The landmark breakthrough in glioblastoma therapy may be in the near future.

\section{Acknowledgments}

The authors would to thank the Pacific Neuroscience Institute for making this work possible.

Funding: None.

\section{Footnote}

Provenance and Peer Review: This article was commissioned by the Guest Editor (Jon Glass) for the series "Targeted systemic therapies for primary and metastatic brain tumors" published in Chinese Clinical Oncology. The article has undergone external peer review.

Conflicts of Interest: All authors have completed the ICMJE uniform disclosure form (available at http://dx.doi. org/10.21037/cco-20-124). The series "Targeted systemic therapies for primary and metastatic brain tumors" was commissioned by the editorial office without any funding or 
sponsorship. The authors have no other conflicts of interest to declare.

Ethical Statement: The authors are accountable for all aspects of the work in ensuring that questions related to the accuracy or integrity of any part of the work are appropriately investigated and resolved.

Open Access Statement: This is an Open Access article distributed in accordance with the Creative Commons Attribution-NonCommercial-NoDerivs 4.0 International License (CC BY-NC-ND 4.0), which permits the noncommercial replication and distribution of the article with the strict proviso that no changes or edits are made and the original work is properly cited (including links to both the formal publication through the relevant DOI and the license). See: https://creativecommons.org/licenses/by-nc-nd/4.0/.

\section{References}

1. Stupp R, Mason WP, van den Bent MJ, et al. Radiotherapy plus Concomitant and Adjuvant Temozolomide for Glioblastoma. N Engl J Med 2005;352:987-96.

2. Stupp R, Taillibert S, Kanner AA, et al. Maintenance Therapy With Tumor-Treating Fields Plus Temozolomide vs Temozolomide Alone for Glioblastoma: A Randomized Clinical Trial. JAMA 2015;314:2535-43.

3. Mischel PS, Cloughesy TF. Targeted molecular therapy of GBM. Brain Pathol 2003;13:52-61.

4. Cancer Genome Atlas Research Network. Comprehensive genomic characterization defines human glioblastoma genes and core pathways. Nature 2008;455:1061-8.

5. Ekstrand AJ, Sugawa N, James CD, et al. Amplified and rearranged epidermal growth factor receptor genes in human glioblastomas reveal deletions of sequences encoding portions of the $\mathrm{N}$ - and/or C-terminal tails. Proc Natl Acad Sci U S A 1992;89:4309-13.

6. Frederick L, Wang XY, Eley G, et al. Diversity and frequency of epidermal growth factor receptor mutations in human glioblastomas. Cancer Res 2000;60:1383-7.

7. Hayashi Y, Ueki K, Waha A, et al. Association of EGFR gene amplification and CDKN2 (p16/MTS1) gene deletion in glioblastoma multiforme. Brain Pathol 1997;7:871-5.

8. Kleihues P, Ohgaki H. Primary and secondary glioblastomas: from concept to clinical diagnosis. Neuro Oncol 1999; 1:44-51.

9. Kuan CT, Wikstrand CJ, Bigner DD. EGF mutant receptor vIII as a molecular target in cancer therapy. Endocr Relat Cancer 2001;8:83-96.

10. Nagane M, Lin H, Cavenee WK, et al. Aberrant receptor signaling in human malignant gliomas: mechanisms and therapeutic implications. Cancer Lett 2001;162 Suppl:S17-S21.

11. Nishikawa R, Ji XD, Harmon RC, et al. A mutant epidermal growth factor receptor common in human glioma confers enhanced tumorigenicity. Proc Natl Acad Sci U S A 1994;91:7727-31.

12. Smith JS, Tachibana I, Passe SM, et al. PTEN mutation, EGFR amplification, and outcome in patients with anaplastic astrocytoma and glioblastoma multiforme. J Natl Cancer Inst 2001;93:1246-56.

13. Watanabe K, Tachibana O, Sata K, et al. Overexpression of the EGF receptor and p53 mutations are mutually exclusive in the evolution of primary and secondary glioblastomas. Brain Pathol 1996;6:217-23; discussion 223-4.

14. Wikstrand CJ, Reist CJ, Archer GE, et al. The class III variant of the epidermal growth factor receptor (EGFRvIII): characterization and utilization as an immunotherapeutic target. J Neurovirol 1998;4:148-58.

15. Vivanco I, Sawyers CL. The phosphatidylinositol 3-Kinase AKT pathway in human cancer. Nat Rev Cancer 2002;2:489-501.

16. Liu W, James CD, Frederick L, et al. PTEN/MMAC1 mutations and EGFR amplification in glioblastomas. Cancer Res 1997;57:5254-7.

17. Schmidt EE, Ichimura K, Goike HM, et al. Mutational profile of the PTEN gene in primary human astrocytic tumors and cultivated xenografts. J Neuropathol Exp Neurol 1999;58:1170-83.

18. Adjei AA, Davis JN, Bruzek LM, et al. Synergy of the protein farnesyltransferase inhibitor SCH66336 and cisplatin in human cancer cell lines. Clin Cancer Res 2001;7:1438-45.

19. Feldkamp MM, Lala P, Lau N, et al. Expression of activated epidermal growth factor receptors, Rasguanosine triphosphate, and mitogen-activated protein kinase in human glioblastoma multiforme specimens. Neurosurgery 1999;45:1442-53.

20. Bögler O, Huang HJ, Kleihues $P$, et al. The p53 gene and its role in human brain tumors. Glia 1995;15:308-27.

21. Momand J, Zambetti GP, Olson DC, et al. The mdm2 oncogene product forms a complex with the p53 protein and inhibits p53-mediated transactivation. Cell 1992;69:1237-45. 
22. Kamijo T, Weber JD, Zambetti G, et al. Functional and physical interactions of the ARF tumor suppressor with p53 and Mdm2. Proc Natl Acad Sci U S A 1998;95:8292-7.

23. Pomerantz J, Schreiber-Agus N, Liegeois NJ, et al. The Ink4a tumor suppressor gene product, p19Arf, interacts with MDM2 and neutralizes MDM2's inhibition of p53. Cell 1998;92:713-23.

24. Mercer WE, Shields MT, Amin M, et al. Negative growth regulation in a glioblastoma tumor cell line that conditionally expresses human wild-type p53. Proc Natl Acad Sci U S A 1990;87:6166-70.

25. Ohgaki H, Kleihues P. Genetic Pathways to Primary and Secondary Glioblastoma. Am J Pathol 2007;170:1445-53.

26. Sherr CJ, Roberts JM. CDK inhibitors: positive and negative regulators of $\mathrm{G} 1$-phase progression. Genes Dev 1999;13:1501-12.

27. Carlson MR, Pope WB, Horvath S, et al. Relationship between survival and edema in malignant gliomas: role of vascular endothelial growth factor and neuronal pentraxin 2. Clin Cancer Res 2007;13:2592-8.

28. Chinot OL, Wick W, Mason W, et al. Bevacizumab plus radiotherapy-temozolomide for newly diagnosed glioblastoma. N Engl J Med 2014;370:709-22.

29. Dai S, Jia R, Zhang X, et al. The PD-1/PD-Ls pathway and autoimmune diseases. Cell Immunol 2014;290:72-9.

30. Sharpe AH, Wherry EJ, Ahmed R, et al. The function of programmed cell death 1 and its ligands in regulating autoimmunity and infection. Nat Immunol 2007;8:239-45.

31. Twyman-Saint Victor C, Rech AJ, Maity A, et al. Radiation and dual checkpoint blockade activate non-redundant immune mechanisms in cancer. Nature 2015;520:373-7.

32. Weber JS, D'Angelo SP, Minor D, et al. Nivolumab versus chemotherapy in patients with advanced melanoma who progressed after anti-CTLA-4 treatment (CheckMate 037): a randomised, controlled, open-label, phase 3 trial. Lancet Oncol 2015;16:375-84.

33. Taube JM, Klein A, Brahmer JR, et al. Association of PD1, PD-1 ligands, and other features of the tumor immune microenvironment with response to anti-PD-1 therapy. Clin Cancer Res 2014;20:5064-74.

34. Nghiem PT, Bhatia S, Lipson EJ, et al. PD-1 Blockade with Pembrolizumab in Advanced Merkel-Cell Carcinoma. N Engl J Med 2016;374:2542-52.

35. Gettinger S, Herbst RS. B7-H1/PD-1 blockade therapy in non-small cell lung cancer: current status and future direction. Cancer J 2014;20:281-9.

36. Berghoff AS, Kiesel B, Widhalm G, et al. Programmed death ligand 1 expression and tumor-infiltrating lymphocytes in glioblastoma. Neuro Oncol 2015;17:1064-75.

37. Nduom EK, Wei J, Yaghi NK, et al. PD-L1 expression and prognostic impact in glioblastoma. Neuro Oncol 2016;18:195-205.

38. Omuro A, Vlahovic G, Lim M, et al. Nivolumab with or without ipilimumab in patients with recurrent glioblastoma: results from exploratory phase I cohorts of CheckMate 143. Neuro Oncol 2018;20:674-86.

39. Filley AC, Henriquez M, Dey M. Recurrent glioma clinical trial, CheckMate-143: the game is not over yet. Oncotarget 2017;8:91779-94.

40. Yovino S, Kleinberg L, Grossman SA, et al. The etiology of treatment-related lymphopenia in patients with malignant gliomas: modeling radiation dose to circulating lymphocytes explains clinical observations and suggests methods of modifying the impact of radiation on immune cells. Cancer Invest 2013;31:140-4.

41. Wherry EJ, Kurachi M. Molecular and cellular insights into T cell exhaustion. Nat Rev Immunol 2015;15:486-99.

42. Reardon DA, Gokhale PC, Klein SR, et al. Glioblastoma Eradication Following Immune Checkpoint Blockade in an Orthotopic, Immunocompetent Model. Cancer Immunol Res 2016;4:124-35.

43. Sakuishi K, Apetoh L, Sullivan JM, et al. Targeting Tim-3 and PD-1 pathways to reverse $T$ cell exhaustion and restore anti-tumor immunity. J Exp Med 2010;207:2187-94.

44. Brennan CW, Verhaak RG, McKenna A, et al. The somatic genomic landscape of glioblastoma. Cell 2013;155:462-77.

45. Furnari FB, Cloughesy TF, Cavenee WK, et al. Heterogeneity of epidermal growth factor receptor signalling networks in glioblastoma. Nat Rev Cancer 2015;15:302-10.

46. Chaffanet $M$, Chauvin C, Laine M, et al. EGF receptor amplification and expression in human brain tumours. Eur J Cancer 1992;28:11-7.

47. Libermann TA, Nusbaum HR, Razon N, et al. Amplification, enhanced expression and possible rearrangement of EGF receptor gene in primary human brain tumours of glial origin. Nature 1985;313:144-7.

48. Libermann TA, Razon N, Bartal AD, et al. Expression of epidermal growth factor receptors in human brain tumors. Cancer Res 1984;44:753-60.

49. Inda MM, Bonavia R, Mukasa A, et al. Tumor heterogeneity is an active process maintained by a mutant EGFR-induced cytokine circuit in glioblastoma. Genes Dev 2010;24:1731-45.

50. Lee SG, Kim K, Kegelman TP, et al. Oncogene AEG-1 
promotes glioma-induced neurodegeneration by increasing glutamate excitotoxicity. Cancer Res 2011;71:6514-23.

51. Callaghan T, Antczak M, Flickinger T, et al. A complete description of the EGF-receptor exon structure: implication in oncogenic activation and domain evolution. Oncogene 1993;8:2939-48.

52. Schuster J, Lai RK, Recht LD, et al. A phase II, multicenter trial of rindopepimut (CDX-110) in newly diagnosed glioblastoma: the ACT III study. Neuro Oncol 2015;17:854-61.

53. Weller M, Kaulich K, Hentschel B, et al. Assessment and prognostic significance of the epidermal growth factor receptor vIII mutation in glioblastoma patients treated with concurrent and adjuvant temozolomide radiochemotherapy. Int J Cancer 2014;134:2437-47.

54. Sampson JH, Heimberger AB, Archer GE, et al. Immunologic escape after prolonged progression-free

Cite this article as: Wagle N, Nguyen M, Carrillo J, Truong J, Dobrawa L, Kesari S. Characterization of molecular pathways for targeting therapy in glioblastoma. Chin Clin Oncol 2020;9(6):77. doi: 10.21037/cco-20-124 survival with epidermal growth factor receptor variant III peptide vaccination in patients with newly diagnosed glioblastoma. J Clin Oncol 2010;28:4722-9.

55. Sampson JH, Aldape KD, Archer GE, et al. Greater chemotherapy-induced lymphopenia enhances tumorspecific immune responses that eliminate EGFRvIIIexpressing tumor cells in patients with glioblastoma. Neuro Oncol 2011;13:324-33.

56. Schuster J, Lai RK, Recht LD, et al. A phase II, multicenter trial of rindopepimut (CDX-110) in newly diagnosed glioblastoma: the ACT III study. Neuro Oncol 2015;17:854-61.

57. Weller M, Butowski N, Tran DD, et al. Rindopepimut with temozolomide for patients with newly diagnosed, EGFRvIII-expressing glioblastoma (ACT IV): a randomised, double-blind, international phase 3 trial. Lancet Oncol 2017;18:1373-85. 